\title{
A Fast and Robust Grid Synchronization Algorithm of a Three-phase Converters under Unbalanced and Distorted Utility Voltages
}

\author{
Kwang-Seob Kim*, Dong-Seok Hyun* and Rae-Yong Kim ${ }^{\dagger}$
}

\begin{abstract}
In this paper, a robust and fast grid synchronization method of a three-phase power converter is proposed. The amplitude and phase information of grid voltages are essential for power converters to be properly connected into the utility. The phase-lock-loop in synchronous reference frame has been widely adopted for the three-phase converter system since it shows a satisfactory performance under balanced grid voltages. However, power converters often operate under abnormal grid conditions, i.e. unbalanced by grid faults and frequency variations, and thus a proper active and reactive power control cannot be guaranteed. The proposed method adopts a second order generalized integrator in synchronous reference frame to detect positive sequence components under unbalanced grid voltages. The proposed method has a fast and robust performance due to its higher gain and frequency adaptive capability. Simulation and experimental results show the verification of the proposed synchronization algorithm and the effectiveness to detect positive sequence voltage.
\end{abstract}

Keywords: Three-phase power converter, Grid synchronization, Phase locked loop(PLL), Enhanced second order generalized integrator(ESOGI)

\section{Introduction}

More and more power converters based on digital control have been spread in a wide range of applications, i.e. motor drives, FACTS, and distributed generation(DG). Especially, climate changes have become worldwide issues and emission of greenhouse gases by fossil fuel such as oil, natural gas, and coal is recognized as major cause of environmental problems. As distributed generation based on renewable energy sources i.e. photovoltaic(PV), wind turbine(WT) are seen as alternatives, they have been increasingly connected to the grid through power converters. This leads to the instability of the utility network and as a consequence, standards for the grid connection of power converters are getting more stringent. Fault ride through (FRT) or low voltage ride through (LVRT) is one of requirements and power converters have to control the active and reactive power without disconnection under faulty grid voltages [1-2].

Grid synchronization is important for a robust operation of power converters which regulate their active and/or reactive power based on positive sequence components under unbalanced grid voltages. Various grid synchronization methods for power converters have been studied.

They are categorized into open-loop and closed-loop methods. Open-loop methods directly estimate phase angle of the voltage based on frame signals. Zero crossing

$\dagger$ Corresponding Author: Dept. of Electrical Engineering, Hanyang University, Korea. (rykim@hanyang.ac.kr)

* Dept. of Electrical Engineering, Hanyang University, Korea (\{kskim, dshyun\}@hanyang.ac.kr)

Received: November 29, 2016; Accepted: January 15, 2017 method is a simple method but it's sensitive grid voltage distortion. In closed-loop methods, the estimation of the phase is adaptively updated through a loop filter controller [3-4].

Phase locked loop technology has been widely used in power converters. Synchronous Reference Frame PLL (SRF-PLL) is a widely adopted technique for synchronizing three-phase systems with the utility [5-6]. Even though the performance of SRF-PLL is satisfactory under balanced grid, its response can be unacceptable under unbalanced, distorted grid voltages [7].

Various method of positive sequence voltage detection has been suggested. All pass filter is used to detect positive sequence components by generating quadrature signal in natural reference frame [8]. A drawback of APF method is short of adaptation of frequency variation. In a EPLL, APF is replaced with EPLL to generate quadrature signal. EPLL is an adaptive notch filter whose frequency moves based on the center frequency of the grid. Decoupled Double synchronous reference frame PLL (DDSRF PLL), Dual SOGI PLL (DSOGI PLL), and Three Phase Enhanced PLL (3phEPLL PLL) are also presented and their performances are analyzed comparatively. These methods show satisfactory performance under faulty grid conditions [9-12].

This paper suggests a fast and robust grid synchronization algorithm of a three phase converter under unbalanced and distorted grid voltages. The proposed algorithm adopts enhanced second order generalized integrator(ESOGI) in synchronous reference frame to detect positive sequence components. It has a high immunity to frequency variation due to the frequency adaptive characteristic of SOGI and a 
high rejection of harmonics in the grid voltage [13].

\section{Conventional SRF-PLL}

\subsection{Phase locked loop(PLL)}

PLL consists of phase detector(PD), loop filter(LF), and voltage controlled oscillator(VCO) as shown in Fig. 1.

PD generates its dc output proportional to the phase difference between its two input signals. It also contains not only dc component but also high frequency $\mathrm{AC}$ components.

LF filters out high frequency $\mathrm{AC}$ components from the output of PD and adopt usually low pass filter(LPF) or proportional-integrator (PI) controller. VCO generates its $\mathrm{AC}$ reference signal as a function of its input voltage.

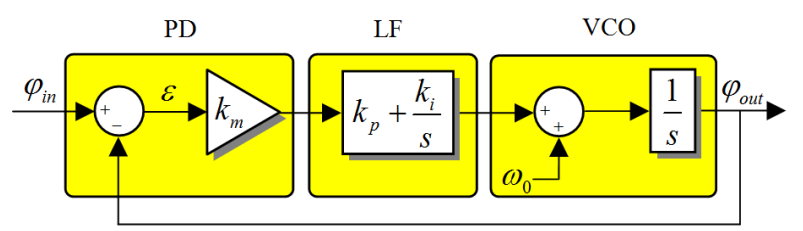

Fig. 1. Basic diagram of PLL

Transfer function of PLL can be written as Eq. (1) where gain of PD and VCO is unit.

$$
G_{P L L}(s)=\frac{\phi_{\text {out }}}{\phi_{\text {in }}}=\frac{2 \zeta \omega_{n} s+\omega_{n}^{2}}{s^{2}+2 \zeta \omega_{n} s+\omega_{n}^{2}}
$$

PLL can be tuned as function of the damping ratio and of the cut-off frequency or the settling time as Eq. (2).

$$
k_{p}=\frac{2 \zeta \omega_{n}}{k_{m}}, \quad k_{i}=\frac{\omega_{n}^{2}}{k_{m}}
$$

\subsection{Three phase PLL system under balanced grid voltage}

Three phase voltages are described in natural reference frame as Eq. (3) under balanced grid voltages.

$$
\left[\begin{array}{l}
v_{a} \\
v_{b} \\
v_{c}
\end{array}\right]=V_{m}\left[\begin{array}{l}
\cos (\omega t) \\
\cos (\omega t-2 \pi / 3) \\
\cos (\omega t-4 \pi / 3)
\end{array}\right]
$$

Then, it can be written in a stationary reference frame according to Eq. (4) and is represented in Eq. (5).

$$
\left[\begin{array}{l}
v_{\alpha} \\
v_{\beta}
\end{array}\right]=\frac{2}{3}\left[\begin{array}{ccc}
1 & -1 / 2 & -1 / 2 \\
0 & \sqrt{3} / 2 & -\sqrt{3} / 2
\end{array}\right] \cdot\left[\begin{array}{c}
v_{a} \\
v_{b} \\
v_{c}
\end{array}\right]
$$

$$
\left[\begin{array}{l}
v_{\alpha} \\
v_{\beta}
\end{array}\right]=V_{m}\left[\begin{array}{l}
\cos (\omega t) \\
\sin (\omega t)
\end{array}\right]
$$

Voltage vector in a stationary reference frame is transformed into SRF by Eq. (6) and is written as Eq. (7).

$$
\begin{gathered}
{\left[\begin{array}{l}
v_{d} \\
v_{q}
\end{array}\right]=\left[\begin{array}{cc}
\cos \hat{\theta} & \sin \hat{\theta} \\
-\sin \hat{\theta} & \cos \hat{\theta}
\end{array}\right] \cdot\left[\begin{array}{l}
v_{\alpha} \\
v_{\beta}
\end{array}\right]} \\
{\left[\begin{array}{l}
v_{d} \\
v_{q}
\end{array}\right]=V_{m}\left[\begin{array}{l}
\cos (\hat{\theta}-\omega t) \\
-\sin (\hat{\theta}-\omega t)
\end{array}\right]}
\end{gathered}
$$

Voltage vector in a stationary reference frame and a synchronous reference frame is shown in Fig. 2.

Rotating voltage vector can be aligned with d-axis or qaxis. When voltage vector is aligned with q-axis, phase difference can be calculated based on Eq. (7) and it can be simplified to Eq. (8) as a phase difference is small enough.

$$
V_{m} \theta_{\text {err }}=V_{m} \cos (\hat{\theta}-\omega t) \approx V_{m} \cdot\left(\frac{\pi}{2}-\omega t+\hat{\theta}\right)
$$

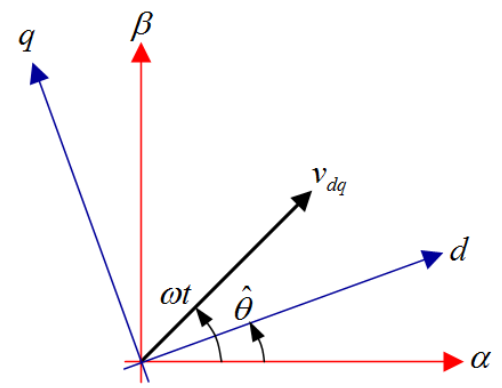

Fig. 2. Rotating voltage vector

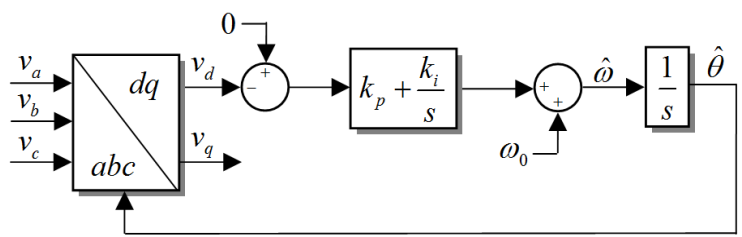

(a)

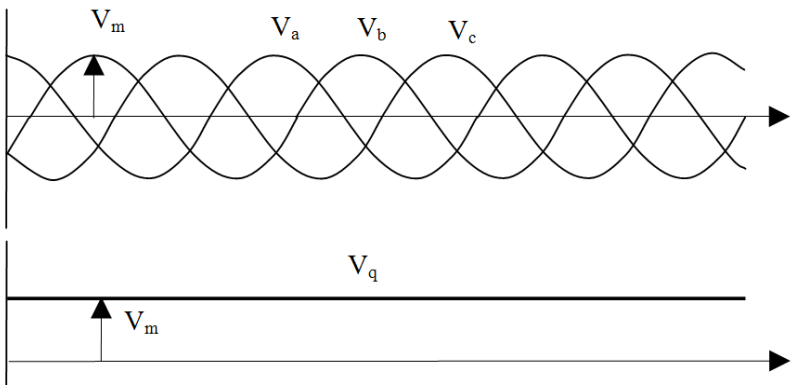

(b)

Fig. 3. Three phase PLL system in SRF, (a) block diagram, (b) voltage vector in SRF 
As voltage vector coincides with d-axis or q-axis, dqaxis values are represented as Eq. (9) and Eq. (10) respectively.

$$
\begin{aligned}
& v_{d}=V_{m}, \quad v_{q}=0 \\
& v_{d}=0, \quad v_{q}=V_{m}
\end{aligned}
$$

Three phase PLL system in synchronous reference frame(SRF) is shown in Fig. 3(a). Rotating vector aligned with q-axis in SRF appears dc components in Fig. 3(b).

\subsection{Behavior under unbalanced grid voltages}

Unbalanced voltage contains positive sequence component, negative sequence component, and zero sequence components expressed in Eq. (11).

$$
\begin{aligned}
& {\left[\begin{array}{l}
v_{a} \\
v_{b} \\
v_{c}
\end{array}\right]=V_{m}^{+} } {\left[\begin{array}{l}
\cos (\omega t) \\
\cos (\omega t-2 \pi / 3) \\
\cos (\omega t-4 \pi / 3)
\end{array}\right]+} \\
& V_{m}^{-}\left[\begin{array}{l}
\cos (\omega t) \\
\cos (\omega t-4 \pi / 3) \\
\cos (\omega t-2 \pi / 3)
\end{array}\right]+V_{m}^{0}\left[\begin{array}{l}
\cos (\omega t) \\
\cos (\omega t) \\
\cos (\omega t)
\end{array}\right]
\end{aligned}
$$

Eq. (11) is transformed into Eq. (12) in stationary reference frame by applying Eq. (4). Zero sequences no longer exist in stationary reference frame.

$$
\left[\begin{array}{l}
v_{\alpha} \\
v_{\beta}
\end{array}\right]=V_{m}^{+}\left[\begin{array}{c}
\cos (\omega t) \\
\sin (\omega t)
\end{array}\right]+V_{m}^{-}\left[\begin{array}{c}
\cos (\omega t) \\
-\sin (\omega t)
\end{array}\right]
$$

According to Eq. (6), it is transformed into Eq. (13). As voltage vector is aligned to q-axis in SRF, it can be described as Eq. (14). Positive sequence components appear as dc value and negative sequence components as second order harmonics in Eq. (14).

$$
\begin{gathered}
{\left[\begin{array}{l}
v_{d} \\
v_{q}
\end{array}\right]=V_{m}^{+}\left[\begin{array}{c}
\cos (\hat{\theta}-\omega t) \\
-\sin (\hat{\theta}-\omega t)
\end{array}\right]+V_{m}^{-}\left[\begin{array}{c}
\cos (\hat{\theta}+\omega t) \\
-\sin (\hat{\theta}+\omega t)
\end{array}\right]} \\
{\left[\begin{array}{l}
v_{d} \\
v_{q}
\end{array}\right]=V_{m}^{+}\left[\begin{array}{l}
0 \\
1
\end{array}\right]+V_{m}^{-}\left[\begin{array}{l}
\sin (2 \omega t) \\
\cos (2 \omega t)
\end{array}\right]}
\end{gathered}
$$

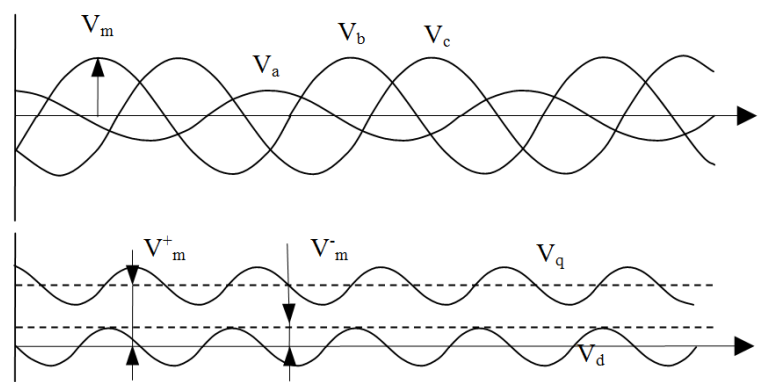

Fig. 4. Voltage vector in SRF under unbalanced grid
According to the analysis, behavior under unbalanced grid voltage where sag in a-phase occurs is depicted in Fig. 4. Due to a negative sequence voltage, second order harmonics appear in SRF. The second order harmonics should be eliminated effectively to secure a proper performance of PLL.

\section{The Proposed Synchronization Method}

\subsection{Second Order Generalized Integrator(SOGI)}

The block diagram using SOGI can be configured as different filters and is shown in Fig. 5.

Band-pass filter(BPF) whose cut-off frequency can be changed adaptively is possible as duplicate output(CD) is selected and its transfer function is Eq. (15) .

$$
G_{D}(s)=\frac{C_{D}(s)}{R(s)}=\frac{k \omega_{i} s}{s^{2}+k \omega_{i} s+\omega_{i}^{2}}
$$

Quadrature signal generator(QSG) is selected by choosing quadrature signal(CQ) which is 90 degree delayed at its cut-off frequency. Eq. (16) describes transfer function of QSG.

$$
G_{Q}(s)=\frac{C_{Q}(s)}{R(s)}=\frac{k \omega_{i}^{2}}{s^{2}+k \omega_{i} s+\omega_{i}^{2}}
$$

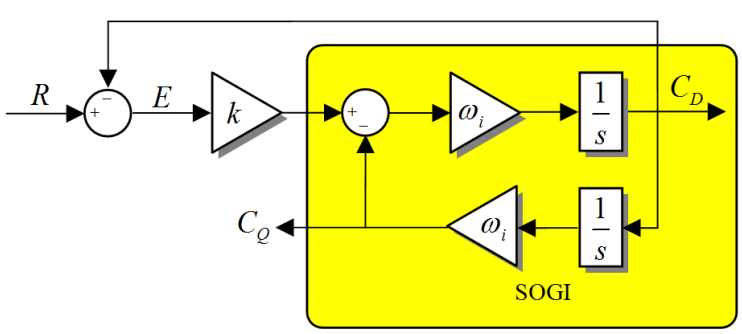

Fig. 5. Basic block diagram of SOGI

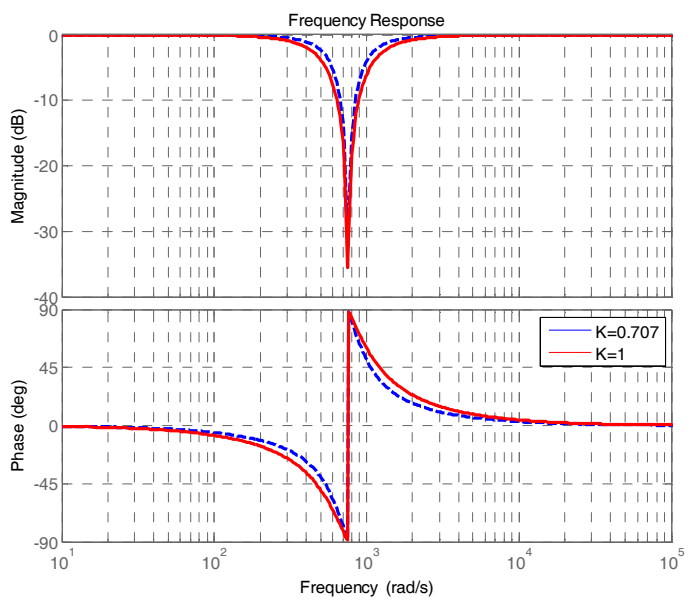

Fig. 6. Bode plot of Eq. (17) 
Adaptive notch filter(ANF) can be accomplished by choosing error signal as output and its transfer function is written as Eq. (17).

$$
G_{E}(s)=\frac{E(s)}{R(s)}=\frac{s^{2}+\omega_{i}^{2}}{s^{2}+k \omega_{i} s+\omega_{i}^{2}}
$$

Bode plot in Fig. 6 represents a frequency response of Eq. (17) of which cut-off frequency is $754[\mathrm{rad} / \mathrm{sec}]$ or 120 [Hz].

Selecting the value of $\mathrm{k}$ requires a trade-off between filtering performance and system dynamics. It is set to 1 in this paper. In next section, this filter is adopted to remove the negative sequence voltage which appears as second order harmonic in SRF under unbalanced grid.

\subsection{The proposed method based on enhanced SOGI}

The proposed PLL for grid synchronization is depicted in Fig. 7. In this configuration, a conventional SRF-PLL is adopted and additional positive sequence detection block called ESOGI is added in SRF. As analyzed in previous section, positive sequence voltage and negative sequence voltage appear as constant dc and second order harmonic in SRF respectively. So, ESOGI eliminates second order harmonic in SRF.

ESOGI consists of SOGI and integrator as shown in Fig. 8 , where SOGI is configured as adaptive notch filter(ANF) of Eq. (17) to extract second order harmonics, i.e. negative sequence components. Gain is set to Eq. (18). Second order harmonics can be eliminated by setting cut-off frequency of SOGI to two times of estimated angular frequency of PLL adaptively.

$$
\omega_{i}=2 \cdot \hat{\omega}
$$

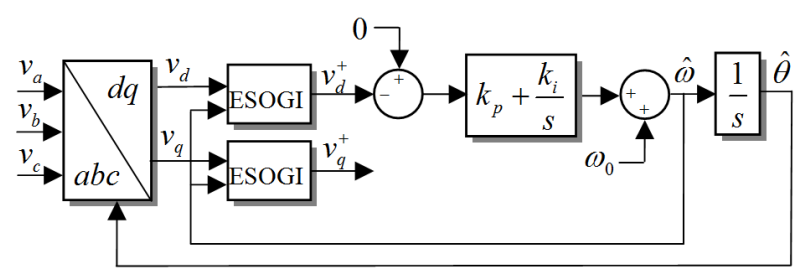

Fig. 7. The proposed PLL using ESOGI

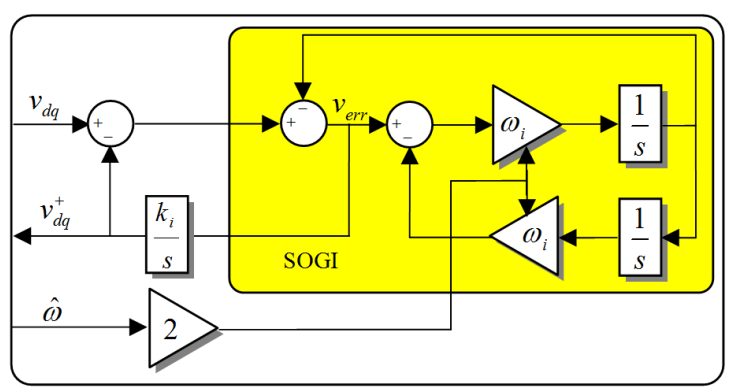

Fig. 8. Enhanced SOGI
Integrator acts as low pass filter with infinite gain for Integrator acts as low pass filter with infinite gain for positive sequence components, i.e. constant dc value. So, dc component of SOGI input is eliminated and only ac components exist in steady state. Integrator gain affects overall performances of the proposed method and it will be analyzed further. Normalized value with respect to fundamental frequency is calculated as Eq. (19).

$$
k_{i}=4 \cdot f_{0}
$$

Second order harmonic of $120[\mathrm{~Hz}]$ sinusoidal signal and/or dc are injected to ESOGI to see a transient time response. Dc component remains and $120[\mathrm{~Hz}]$ harmonic disappears after a period of cycle as shown in Fig. 9.

\subsection{Performance evaluation of the proposed method} (20).

Overall transfer function of ESOGI is represented as Eq.

$$
G_{p}(s)=\frac{v_{d q}^{+}(s)}{v_{d q}(s)}=\frac{k_{i} s^{2}+k_{i} \omega_{i}^{2}}{s^{3}+\left(k_{i}+\omega_{i}\right) s^{2}+\omega_{i}^{2} s+k_{i} \omega_{i}^{2}}
$$

Harmonic reduction ratio of ESOGI can be calculated as Eq. (21) by using Eq. (20).

$$
\left|G_{p}\left(j h \omega_{0}\right)\right|=\frac{k_{i} \cdot\left|\omega_{i}^{2}-\omega_{h+1}^{2}\right|}{\sqrt{\left(\omega_{h+1} \cdot\left(\omega_{i}^{2}-\omega_{h+1}^{2}\right)\right)^{2}+\left(k_{i} \omega_{i}^{2}-\left(k_{i}+\omega_{i}\right) \cdot \omega_{h+1}^{2}\right)^{2}}}
$$

where

$$
\omega_{h+1}=(h+1) \cdot \omega_{0}, \quad h=6 N \pm 1(N=1,2,3, \cdots)
$$

Harmonics of 5 th and 7 th is common in a real grid and they appear as 6th and 7th harmonics respectively in a positive sequence SRF. Simulation results are shown in Fig. 10. Harmonics of 6th and 8th in SRF is reduced down to $9.7 \%$ and $7.5 \%$ respectively. Simulation results are exactly same to calculated value based on Eq. (21).

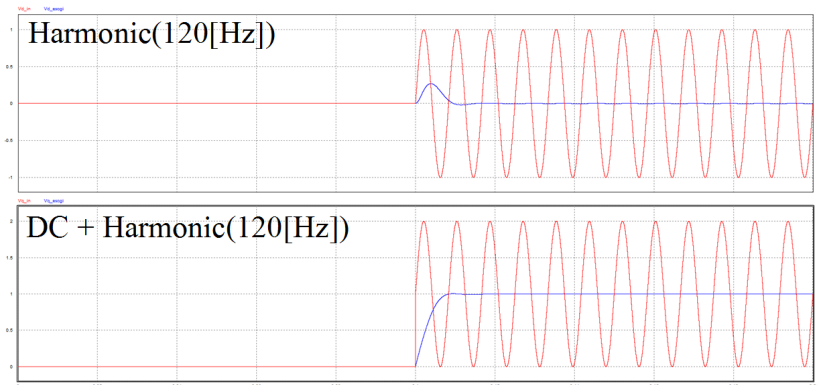

Fig. 9. Transient time response of ESOGI to second order harmonic input 


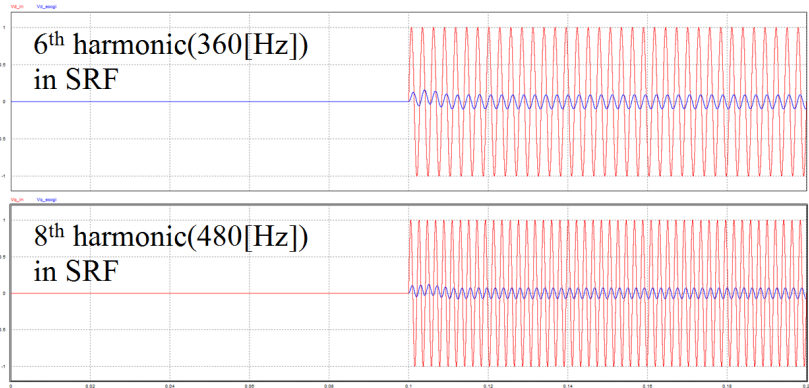

Fig. 10. Harmonic characteristics of ESOGI

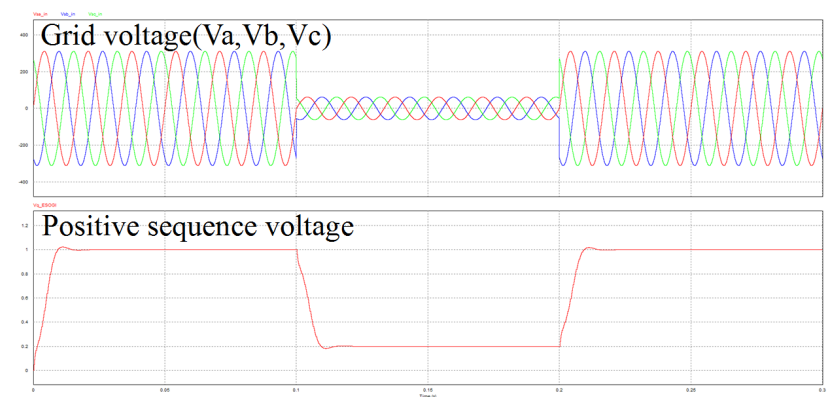

(a)
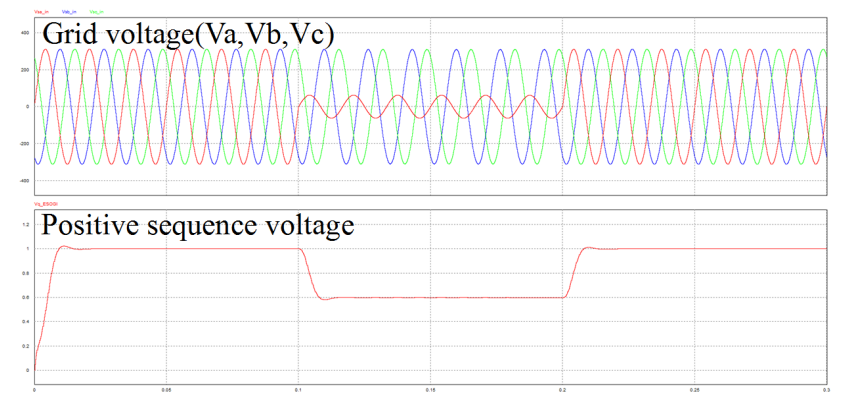

(b)

Fig. 11. Positive sequence voltage, (a) under symmetric grid fault of $20 \%$, (b) under single phase ground fault of $20 \%$

\section{Simulation results}

Simulations using PSIM are carried out to verify the effectiveness of the proposed method under various grid conditions. Digital controller is implemented with sampling frequency of $6[\mathrm{kHz}]$. Line voltage is $380 \mathrm{Vrms}$ and controller is based on per unit.

LF gains are calculated according to Eq. (2) where damping $\operatorname{ratio}(\zeta)$ is 0.707 and cut-off frequency $\left(\omega_{n}\right)$ is $62.8[\mathrm{rad} / \mathrm{sec}]$. Proportional gain $\left(K_{p}\right)$ is 89 and integrator gain $\left(K_{i}\right)$ is 3948 .

\subsection{Voltage sag under grid faults}

Grid voltage sags that affect converters connected to the utility are categorized into four types. Type A is a

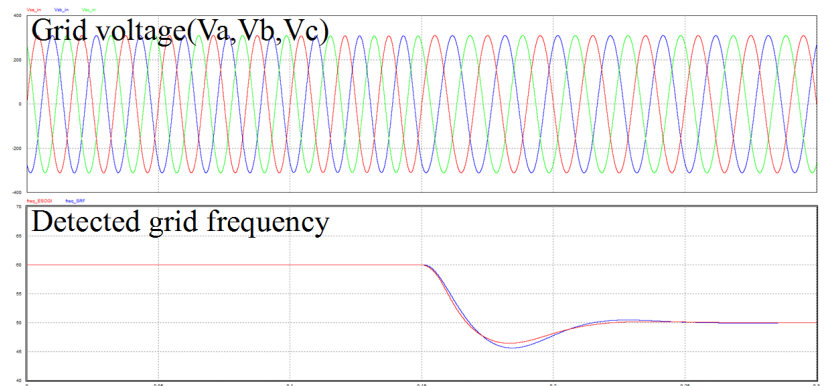

Fig. 12. Simulation waveforms under frequency jump

symmetric grid fault as consequence of a three phase faults and gives rise to a balanced voltage drop. There are only positive sequence voltages under a balanced voltage. Others are asymmetric grid faults which generate unbalanced grid voltages. Type ' $\mathrm{B}$ ' has a zero sequence voltage. Type ' $\mathrm{C}$ ' and type 'D' caused by a phase to ground or a phase to phase short circuit at the primary side of transformer have not only positive sequence voltages but also negative sequence voltages.

Simulation waveforms of type ' $A$ ' and type ' $B$ ' are shown in Fig. 11(a) and (b) respectively. There is no second order harmonics so that the proposed method detects a positive sequence voltage effectively by eliminating second order harmonics under both balanced grid and unbalanced grid.

\subsection{Grid frequency jump}

Extreme frequency variation of a positive sequence voltage from $60 \mathrm{~Hz}$ to $50 \mathrm{~Hz}$ was applied to analyze the frequency adaptive response. Even though frequency changes dramatically, it detects successfully and simulation waveform is shown in Fig. 12.

\section{Experiment}

Experiment is carried out to verify the proposed method as shown in Fig. 13. Digital controller adopts a high performance CPU of TMS320C28346 and its sampling frequency is $6[\mathrm{kHz}]$.

Experimental waveforms are shown in Fig. 14 under unbalanced grid voltages. Three phase voltages are decreased down to $20 \%$ of their nominal voltage of $380 \mathrm{VLL}$ shown in Fig. 14(a) when symmetric grid fault occurs. Proposed method detects positive sequence voltage within one cycle.

As single phase to ground fault occurs, one phase voltage goes down to $20 \%$ of its nominal voltage as in Fig. 14(b). According as previous analysis and simulation, unbalanced grid voltage is composed of a positive sequence voltage, a negative sequence voltage, and a zero sequence voltage. A positive sequence voltage and a negative sequence voltage appear as dc and second order harmonics in SRF. The 


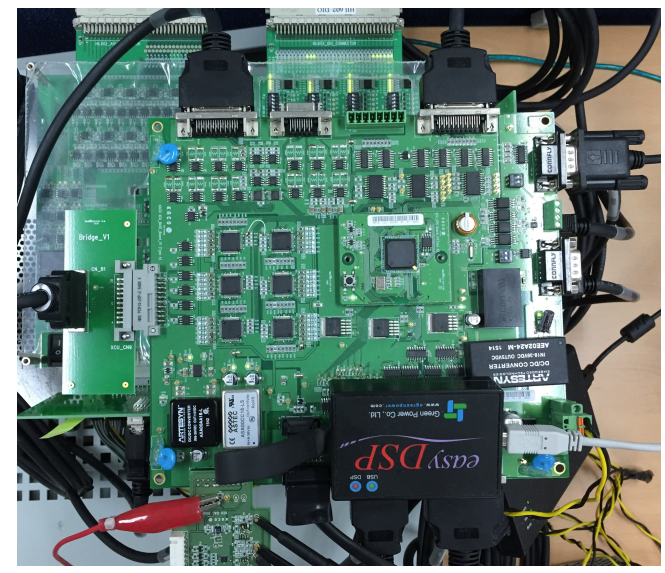

Fig. 13. Experimental set-up

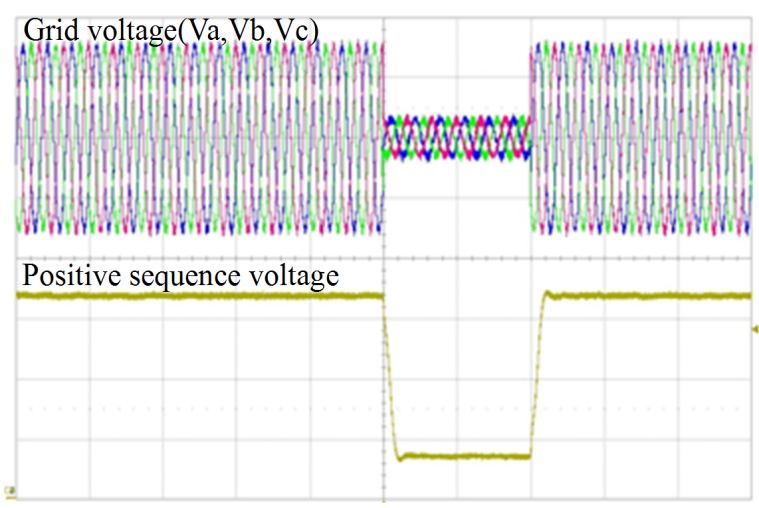

(a)

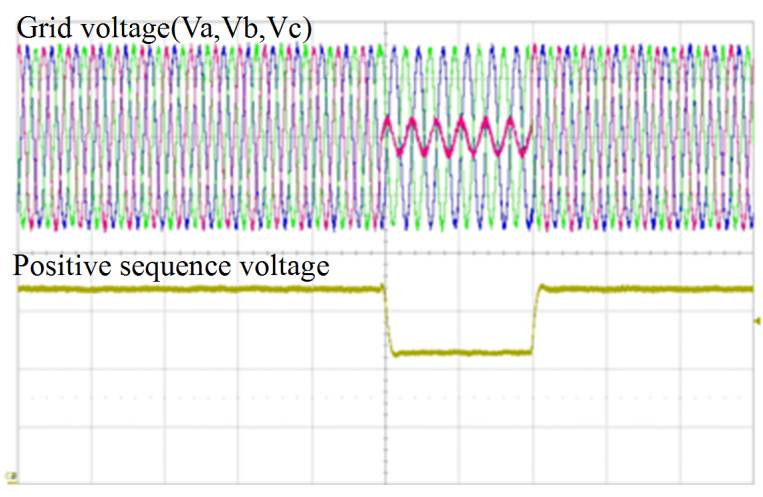

(b)

Fig. 14. Positive sequence voltage(q-axis voltage in SRF), (a) under symmetric grid fault of $20 \%$, (b) under single phase ground fault of $20 \%$, upper : 3 phase grid voltage(200[V/div]), lower : positive sequence voltage $0.3[\mathrm{pu} / \mathrm{div}], 50[\mathrm{msec} / \mathrm{div}]$

proposed ESOGI filters out the second order harmonics caused by negative sequence voltages in SRF so that only positive sequence voltage appear.

Grid frequency is changed from $60 \mathrm{~Hz}$ to $50 \mathrm{~Hz}$ to see clearly how well to track frequency jump. Waveforms in Fig. 15 show PLL tracks frequency variation exactly.

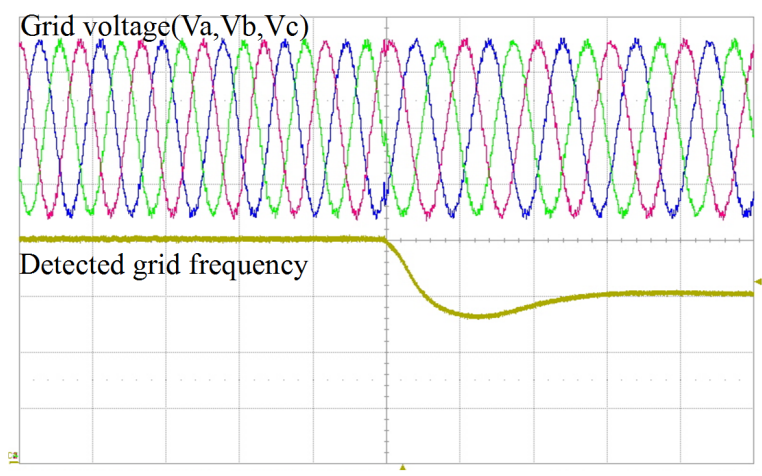

Fig. 15. Experimental waveforms under frequency jump, upper : 3 phase grid voltage(200[V/div]), lower : estimated frequency $([10 \mathrm{~Hz} / \mathrm{div}]), 20[\mathrm{msec} / \mathrm{div}]$

\section{Conclusion}

A new grid synchronization method of a three phase converter is presented. It adopts ESOGI in SRF to detect a positive sequence voltage where ESOGI is composed of SOGI and integrator. SOGI is configured as an adaptive notch filter whose gain is adjusted to two times angular frequency of positive sequence voltage. Integrator gives rise to a smooth transient response and reduces harmonics in proportional to its order.

Simulation and experiment are carried out to verify the proposed grid synchronization method. Results show the proposed method detects a positive sequence voltage under unbalanced and distorted grid voltages in a fast and robust way. So, this method can be adopted in a wide range of three phase power converter applications.

\section{References}

[1] F. Blaabjerg, R. Teodorescu, M. Liserre, A.V. Timbus, "Overview of Control and Grid Synchronization for Distributed Power Generation Systems," IEEE Trans. on Industrial Electronics, vol. 53, no. 5, pp. 13981409, Oct 2006.

[2] L. Zhang and M. Bollen, "Characteristic of voltage dips (sags) in power systems," Power Delivery, IEEE Transactions on, vol. 15, no. 2, pp. 827-832, 2000.

[3] A. Timbus, M. Liserre, R. Teodorescu, and F. Blaabjerg, "Synchronization methods for three phase distributed power generation systems. an overview and evaluation," in Power Electronics Specialists, 2005 IEEE 36th Conference on, June 12, 2005, pp. 2474-2481.

[4] R. M. Santos Filho, P. F. Seixas, P. C. Cortizo, L. A. B. Torres, A. F. Souza, "Comparison of Three SinglePhase PLL Algorithms for UPS Applications," IEEE Trans. on Industrial Electronics, vol. 55, no. 8, pp. 2923-2932, August 2008. 
[5] L. Arruda, S. Silva, and B. Filho, "PLL structures for utility connected systems," in Industry Applications Conference, 2001. Thirty-Sixth IAS Annual Meeting. Conference Record of the 2001 IEEE, vol. 4, 30 Sept.-4 Oct. 2001, pp. 2655-2660vol.4.

[6] S.-K. Chung, "A phase tracking system for three phase utility interface inverters," Power Electronics, IEEE Transactions on, vol. 15, no. 3, pp. 431-438, May 2000.

[7] V. Kaura and V. Blasko, "Operation of a phase locked loop system under distorted utility conditions," in Applied Power Electronics Conference and Exposition, 1996. APEC '96. Conference Proceedings 1996., Eleventh Annual, vol. 2, 3-7 March 1996, pp. 703708, vol. 2.

[8] S. Lee, J. Kang, and S. Sul, "A new phase detection method for power conversion systems considering distorted conditions in power system" in Proc. IEEEIAS Annu.Meeting, 1999, vol. 4, pp. 2167-2172.

[9] M. Karimi-Ghartemani and M. Iravani, "A method for synchronization of power electronic converters in polluted and variable-frequency environments," Power Systems, IEEE Transactions on, vol. 19, no. 3, pp. 1263-1270, 2004.

[10] P. Rodriguez, R. Teodorescu, I. Candela, A. Timbus, M. Liserre, and F. Blaabjerg, "New positive sequence voltage detector for grid synchronization of power converters under faulty grid conditions," in Power Electronics Specialists, 2006 IEEE 37th Conference on, 2006, pp. 1492-1498.

[11] P. Rodriguez, J. Pou, J. Bergas, I. Candela, R. Burgos, and D. Boroyevic, "Double synchronous reference frame PLL for power converters control," in Power Electronics Specialists, 2005 IEEE 36th Conference on, 2005, pp. 1415-1421.

[12] Rodriguez, P.; Pou, J.; Bergas, J.; Candela, J.I.; Burgos, R.P.; Boroyevich, D., "Decoupled Double Synchronous Reference Frame PLL for Power Converters Control," Power Electronics, IEEE Transactions on, vol. 22, no. 2, pp. 584-592, March 2007.

[13] X. Yuan, W. Merk, H. Stemmler, and J. Allmeling, "Stationary-frame generalized integrators for current control of active power filters with zero steadystate error for current harmonics of concern under unbalanced and distorted operating conditions," Industry Applications, IEEE Transactions on, vol. 38, no. 2, pp. 523-532, March-April 2002.

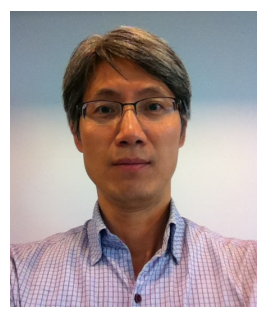

Kwang-Seob Kim He received B.S and M.S degree in electrical engineering from Hanyang University in 1994 and 1996 respectively. His research interests are high power converter, $\mathrm{AC}$ drive system, robust control, and distributed energy resource.

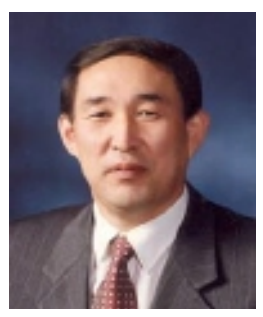

Dong-Seok Hyun He received the B.S. and M.S. degrees from Hanyang University, Seoul, Korea, in 1973 and 1978, respectively, and the Ph.D. degree from Seoul National University, Seoul, Korea, in 1986, all in electrical engineering. From 1976 to 1979 , he was a Researcher with the Agency of Defense Development, Korea. From 1984 to 1985, he was a Research Associate in the Department of Electrical Engineering, University of Toledo, Toledo, $\mathrm{OH}$, and from 1988 to 1989, he was a Visiting Professor in the Department of Electrical Engineering, Technical University of Munich, Germany. Since 1979, he has been with Hanyang University, where he is currently a Professor in the Department of Electrical Engineering. He is the author of more than 650 publications concerning electric machine design, highpower engineering, power electronics, and motor drives. His research interests include power electronics, motor drives, traction, and their control systems. Dr. Hyun is a member of the IEEE Power Electronics, Industrial Electronics, Industry Applications, and Electron Devices Societies. He is also a member of the Institution of Engineering and Technology, the Korean Institute of Power Electronics, and the Korean Institute of Electrical Engineers.

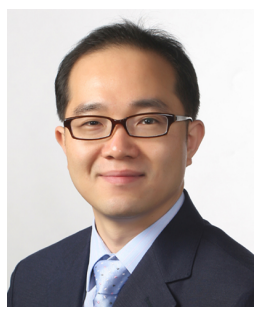

Rae-Young Kim He received the B.S. and M.S. degrees from Hanyang University, Seoul, Korea, in 1997 and 1999, respectively, and the Ph.D. degree from Virginia Polytechnic Institute and State University, Blacksburg, VA, USA, in 2009, all in electrical engineering. From 1999 to 2004, he was a Senior Researcher at the Hyosung Heavy Industry R\&D Center, Seoul, Korea. In 2009, he was a Postdoctoral Researcher at National Semiconductor Corporation, Santa Clara, CA, USA, involved in a smart home energy management system. Since 2010, he has been with Hanyang University, where he is currently an Assistant Professor in the Department of Electrical and Biomedical Engineering. His research interests include modeling and control of power converter systems, soft-switching techniques, energy management systems in smart grid applications, power converter systems for renewable energies, and motor drive systems. Dr. Kim has been a member of the IEEE IAS Industry Power Converters (IAS) Industrial Power Converter Committee since 2009 and also served as a Reviewer for the IEEE TRANSACTION ON INDUSTRIAL ELECTRONICS and the IEEE TRANSACTION ON INDUSTRY APPLICATIONS. He was a recipient of the 2007 First Prize Paper Award from the IEEE IAS. 